\title{
ANALYTIC FUNCTIONS, IDEALS, AND DERIVATION RANGES ${ }^{1}$
}

\author{
R. E. WEBER
}

\begin{abstract}
When $A$ is in the Banach algebra $\mathscr{H}(\mathscr{H})$ of all bounded linear operators on a Hilbert space $\mathscr{H}$, the derivation generated by $A$ is the bounded operator $\Delta_{A}$ on $\mathscr{B}(\mathscr{H})$ defined by $\Delta_{A}(X)=A X-X A$. It is shown that (i) if $B$ is an analytic function of $A$, then the range of $\Delta_{B}$ is contained in the range of $\Delta_{A}$; (ii) if $U$ is a nonunitary isometry, then the range of $\Delta_{l}$. contains nonzero left ideals; (iii) if $U$ and $V$ are isometries with orthogonally complemented ranges, then the span of the ranges of the corresponding derivations is all of $\mathscr{B}(\mathscr{H})$.
\end{abstract}

1. It follows from the elementary properties of derivations that the set of all $B$ such that $\mathscr{R}\left(\Delta_{13}\right) \subset \mathscr{R}\left(\Delta_{A}\right)$ is a subalgebra of $\mathscr{B}(\mathscr{H})$. (See $\left[9\right.$, p. 4].) Therefore if $B$ is a polynomial in $A$, then $\mathscr{R}\left(\Delta_{13}\right) \subset \mathscr{R}\left(\Delta_{A}\right)$. We will generalize this to analytic functions. In the following, $\mathscr{H}$ denotes a separable complex Hilbert space.

THEOREM 1. Let $A \in \mathscr{B}(\mathscr{H})$ and let $f(z)$ be a function analytic on an open set containing $\sigma(A)$. If $B=f(A)$, then $\mathscr{R}\left(\Delta_{1}\right) \subset \mathscr{R}\left(\Delta_{A}\right)$.

For the proof we need the following result on analytic functions of commuting operators.

Let $\mathscr{A}$ be a commutative Banach algebra with maximal ideal space $\mathscr{M}_{\mathscr{S}}$ and let $a_{1}$ and $a_{2}$ belong to $\mathscr{A}$. The joint spectrum of $a_{1}$ and $a_{2}$ is the set $\left\{\left(\varphi\left(a_{1}\right), \varphi\left(a_{2}\right)\right): \varphi \in \mathscr{M}_{.}\right\}$and is denoted by $\sigma\left(a_{1}, a_{2}\right)$. (See Gamelin $[3$, p. 76] for a discussion of the joint spectrum and the proof of the following lemma.)

LEMMA 1. There exists a unique rule assigning to every ordered pair $\left(a_{1}, a_{2}\right)$ of elements in $\mathscr{A}$ and to every complex valued function of two complex variables $f\left(z, w^{\prime}\right)$ analytic in a neighborhood of $\sigma\left(a_{1}, a_{2}\right)$, an element

Received by the editors October 16, 1972 and, in revised form, January 10, 1973.

AMS (MOS) subject classifications (1970). Primary 47B47; Secondary 47A50.

Key words and phrases. Derivation ranges, left ideals, analytic functions, orthogonally complemented ranges.

1 This paper contains part of a doctoral dissertation written under the direction of Professor James Williams at Indiana University. 
$f\left(a_{1}, a_{2}\right) \in \mathscr{A}$ satisfying the following conditions:

(a) If $f(z, w)=\sum c_{i} d_{j} z^{i} w^{j}$ is a polynomial, then $f\left(a_{1}, a_{2}\right)=\sum c_{i} d_{j} a_{1}^{i} a_{2}^{j}$.

(b) If $f(z, w)$ and $g(z, w)$ are analytic in a neighborhood of $\sigma\left(a_{1}, a_{2}\right)$, then

and

$$
(f+g)\left(a_{1}, a_{2}\right)=f\left(a_{1}, a_{2}\right)+g\left(a_{1}, a_{2}\right)
$$

$$
(f g)\left(a_{1}, a_{2}\right)=f\left(a_{1}, a_{2}\right) g\left(a_{1}, a_{2}\right) .
$$

(c) If $f(z)$ is analytic in a neighborhood $U$ of $\sigma\left(a_{1}\right)$ and if $f_{1}(z, w)$ is the extension of $f(z)$ to $U \times \mathscr{C}$ defined by $f_{1}(z, w)=f(z)$, then $f_{1}\left(a_{1}, a_{2}\right)=f\left(a_{1}\right)$ where $f\left(a_{1}\right)$ is an analytic function of the element $a_{1}$ in the sense of the Riesz-Dunford functional calculus (Dunford and Schwartz [2, p. 566]).

Proof of Theorem 1. For $A \in \mathscr{B}(\mathscr{H})$, let $L_{A}$ and $R_{A}$ be the operators on $\mathscr{B}(\mathscr{H})$ defined by $L_{A}(X)=A X$ and $R_{A}(X)=X A$. It is not difficult to show that $\sigma\left(L_{A}\right)=\sigma\left(R_{A}\right)=\sigma(A)$. Therefore if $f(z)$ is analytic on a neighborhood of $\sigma(A)$, then both $f\left(L_{A}\right)$ and $f\left(R_{A}\right)$ are defined by the usual Riesz-Dunford functional calculus. Furthermore, it is known [5, p. 33] that $f\left(L_{A}\right)=L_{f(A)}$ and $f\left(R_{A}\right)=R_{f(A)}$. Let $\mathscr{A}$ be the maximal abelian subalgebra of $\mathscr{B}(\mathscr{B}(\mathscr{H}))$ containing $L_{A}, R_{A}$, and the identity. Then the spectrum of $L_{A}$ (and $R_{A}$ ) with respect to the algebra $\mathscr{A}$ is equal to the spectrum of $L_{A}$ (and $R_{A}$ ) with respect to the algebra $\mathscr{B}(\mathscr{B}(\mathscr{H})$ ) which is $\sigma(A)$. (See [7, p. 34].) We will apply Lemma 1 to the commutative algebra $\mathscr{A}$. If $g(z, w)=(f(z)-f(w)) /(z-w)$, then it can easily be shown that $g(z, w)$ is analytic on a neighborhood of $\sigma\left(L_{A}, R_{A}\right)$. Let $h(z, w)=(z-w) g(z, w)$. Then by Lemma 1 part (b) there exists an operator $h\left(L_{A}, R_{A}\right)$ in $\mathscr{A}$ such that $h\left(L_{A}, R_{A}\right)=f\left(L_{A}\right)-f\left(R_{A}\right)$ and by parts (a) and (b) $h\left(L_{A}, R_{A}\right)=$ $\left(L_{A}-R_{A}\right) g\left(L_{A}, R_{A}\right)$. Therefore $f\left(L_{A}\right)-f\left(R_{A}\right)=\left(L_{A}-R_{A}\right) g\left(L_{A}, R_{A}\right)$. Hence

$$
\Delta_{f(A)}=L_{f(A)}-R_{f(A)}=f\left(L_{A}\right)-f\left(R_{A}\right)=\Delta_{A} g\left(L_{A}, R_{A}\right)
$$

and therefore $\mathscr{R}\left(\Delta_{f(A)}\right) \subset \mathscr{R}\left(\Delta_{A}\right)$.

Corollary 1. Let $A \geqq 0$ be an element of $\mathscr{B}(\mathscr{H})$ with $0 \notin \sigma(A)$. Then $\mathscr{R}\left(\Delta_{A^{1 / 2}}\right)=\mathscr{R}\left(\Delta_{A}\right)$.

Proof. Since the function $f(z)=z^{1 / 2}$ is analytic on the right half plane, $\mathscr{R}\left(\Delta_{A^{1 / 2}}\right) \subset \mathscr{R}\left(\Delta_{A}\right)$. The reverse inclusion follows from the fact that $A=\left(A^{1 / 2}\right)^{2}$.

2. Stampfli [8] has shown that the range of a derivation does not contain any nonzero two-sided ideals. We will see that the range of a derivation generated by a nonunitary isometry does contain nonzero left ideals. 
REMARK. If $U$ is a pure isometry and $\mathscr{D}=\mathscr{R}(U)^{\perp}$, then it can be shown that all operators of the form

$$
A=\left[\begin{array}{ccccccc}
A_{0} & 0 & 0 & 0 & \cdot & \cdot & \cdot \\
A_{1} & 0 & 0 & 0 & \cdot & \cdot & \cdot \\
A_{2} & 0 & 0 & 0 & \cdot & \cdot & \cdot \\
\cdot & & & & & & \\
\cdot & & & & & & \\
\cdot & & & & & &
\end{array}\right]
$$

on $\mathscr{H}=\mathscr{D} \oplus U(\mathscr{D}) \oplus U^{2}(\mathscr{D}) \oplus \cdots$ are in $\mathscr{R}\left(\Delta_{U}\right)$. (See Pearcy [6] or Halmos [4].) It is an immediate consequence that $\mathscr{B}(\mathscr{H})\left(1-U U^{*}\right) \subset$ $\mathscr{R}\left(\Delta_{U}\right)$. This result can be extended to all isometries.

TheOREM 2. Let $U$ be an isometry on $\mathscr{H}$. If $P=1-U U^{*}$, then $\mathscr{B}(\mathscr{H}) P \subset \mathscr{R}\left(\Delta_{U}\right)$.

Proof. Let $U=V \oplus W$ on $\mathscr{H}=\mathscr{H}_{1} \oplus \mathscr{H}_{2}$ where $V$ is a pure isometry, $W$ is a unitary, and $\mathscr{H}_{i}$ is an infinite dimensional Hilbert space for $i=1,2$. Given $X \in \mathscr{B}(\mathscr{H})$ where

$$
X=\left[\begin{array}{ll}
X_{1} & X_{2} \\
X_{3} & X_{4}
\end{array}\right]
$$

choose $Y_{1} \in \mathscr{B}\left(\mathscr{H}_{1}\right)$ such that $X_{1}\left(1-V V^{*}\right)=V Y_{1}-Y_{1} V$ (the existence of which is guaranteed by the above remark). If we let

$$
Y=\left[\begin{array}{cc}
Y_{1} & 0 \\
W^{*} X_{3}\left(1-V V^{*}\right) & 0
\end{array}\right]
$$

then a computation shows that $X\left(1-U U^{*}\right)=\Delta_{U}(Y)$.

REMARKS. (1) A more algebraic proof can be obtained by seeing that for $Y \in \mathscr{B}(\mathscr{H})$, the operator $X=\sum_{k=0}^{\infty} U^{k} P Y P U^{* k+1}$ is bounded and that $\Delta_{U}\left(U^{*} Y P-X\right)=Y P$.

(2) Let $U=V \oplus W$ on $\mathscr{H}=\mathscr{H}_{1} \oplus \mathscr{H}_{2}$ where $V$ and $W$ are both required only to be isometries. For $Y=\left(Y_{i}\right) \in \mathscr{B}(\mathscr{H})$

$$
\Delta_{U}(Y)=\left[\begin{array}{cc}
V Y_{1}-Y_{1} V & V Y_{2}-Y_{2} W \\
W Y_{3}-Y_{3} V & W Y_{4}-Y_{4} W
\end{array}\right]
$$

and for $X=\left(X_{i}\right) \in \mathscr{B}(\mathscr{H})$

$$
X\left(1-U U^{*}\right)=\left[\begin{array}{ll}
X_{1}\left(1-V V^{*}\right) & X_{2}\left(1-W W^{*}\right) \\
X_{3}\left(1-V V^{*}\right) & X_{4}\left(1-W W^{*}\right)
\end{array}\right] .
$$


Since $U$ is an isometry, $\mathscr{B}(\mathscr{H})\left(1-U U^{*}\right) \subset \mathscr{R}\left(\Delta_{U}\right)$ by Theorem 2 . By considering the $(2,1)$ positions in the above matrices, it follows that given any bounded operator $X: \mathscr{H}_{1} \rightarrow \mathscr{H}_{2}$, there exists a bounded operator $Y: \mathscr{H}_{1} \rightarrow \mathscr{H}_{2}$ such that $X\left(1-V V^{*}\right)=W Y-Y V$. In particular, if $V$ and $W$ are isometries on $\mathscr{H}_{1}$ and $W_{1}$ is a unitary from $\mathscr{H}_{1}$ onto $\mathscr{H}_{2}$, then $V \oplus W_{1} W W_{1}^{*}$ is an isometry on $\mathscr{H}=\mathscr{H}_{1} \oplus \mathscr{H}_{2}$. Therefore, for each $X \in \mathscr{B}\left(\mathscr{H}_{1}\right)$ there exists a $Y \in \mathscr{B}\left(\mathscr{H}_{1}\right)$ such that $W_{1} X\left(1-V V^{*}\right)=$ $W_{1} W W_{1}^{*}\left(W_{1} Y\right)-\left(W_{1} Y\right) V$. Therefore $X\left(1-V V^{*}\right)=W Y-Y V$.

COROLlaRY. If $V$ and $W$ are isometries on $\mathscr{H}$, then $\mathscr{B}(\mathscr{H})\left(1-V V^{*}\right)$ is contained in the range of the intertwining operator $T(X)=W X-X V$.

Remarks. (1) By the use of Theorem 2 we can show that $\mathscr{R}\left(\Delta_{U}\right)$ contains other left ideals, in fact $\mathscr{B}(\mathscr{H})\left(1-U_{\lambda} U_{\lambda}^{*}\right) \subset \mathscr{R}\left(\Delta_{U}\right)$ for $U_{\lambda}=$ $(U-\lambda)(1-\bar{\lambda} U)^{-1}$. To obtain an operator such that its derivation range contains right ideals, we need only consider the adjoint of a nonunitary isometry.

(2) The right ideal generated by $1-U U^{*}$ is not contained in $\mathscr{R}\left(\Delta_{U}\right)$. (See [9].)

3. It was observed by Halmos [4] that every operator on an infinite dimensional Hilbert space is the sum of two commutators. This result can be strengthened.

THEOREM 3. Let $U$ and $V$ be isometries on an infinite dimensional Hilbert space. If $\mathscr{R}(U) \oplus \mathscr{R}(V)=\mathscr{H}$, then $\mathscr{R}\left(\Delta_{U}\right)+\mathscr{R}\left(\Delta_{V}\right)=\mathscr{B}(\mathscr{H})$.

Proof. Let $P_{1}=1-U U^{*}$ and $P_{2}=1-V V^{*}$. Then for $X \in \mathscr{B}(\mathscr{H})$, $X=X P_{1}+X P_{2}$. Hence $X \in \mathscr{R}\left(\Delta_{U}\right)+R\left(\Delta_{V}\right)$ by Theorem 2 .

REMARK. Although Stampfli [8] has shown that $\mathscr{R}\left(\Delta_{A}\right)$ cannot be dense in $\mathscr{B}(\mathscr{H})$, Theorem 3 shows that $\mathscr{R}\left(\Delta_{U}\right)+\mathscr{R}\left(\Delta_{V}\right)$ is dense if $U$ and $V$ are the isometries $U: \mathscr{H} \rightarrow \mathscr{M}$ and $V: \mathscr{H} \rightarrow \mathscr{M}^{\perp}$ associated with any infinite dimensional subspace $\mathscr{M}$ of infinite deficiency.

\section{REFERENCES}

1. J. H. Anderson, Derivations, commutators, and the essential numerical range, Thesis, Indiana University, Bloomington, Ind., 1971.

2. N. Dunford and J. T. Schwartz, Linear operators. I, Pure and Appl. Math., vol. 7, Interscience, New York, 1958. MR 22 \#8302.

3. F. W. Gamelin, Uniform algebras, Prentice-Hall, Englewood Cliffs, N.J., 1969.

4. P. R. Halmos, Commutators of operators. II, Amer. J. Math. 76 (1954), 191-198. MR 15, 538.

5. G. Lumer and M. Rosenblum, Linear operator equations, Proc. Amer. Math. Soc. 10 (1959), 32-41. MR 21 \#2927. 
6. C. Pearcy, On commutators of operators on Hilbert space, Proc. Amer. Math. Soc. 16 (1965), 53-59. MR 30 \#452.

7. C. E. Rickart, General theory of Banach algebras, University series in Higher Math., Van Nostrand, Princeton, N.J., 1960. MR 22 \#5903.

8. J. G. Stampfli, On the range of a derivation (to appear).

9. R. E. Weber, Derivation ranges, Thesis, Indiana University, Bloomington, Ind., 1972.

Department of Mathematics, Indiana University Southeast, Warder Park, JEFFERSONVILLE, INDIANA 47130

Current address: Department of Mathematics, Indiana University Southeast, New Albany, Indiana 47150 